\title{
Secondary Structure of Bovine Albumin as Studied by Polarization-Sensitive Multiplex CARS Spectroscopy
}

\author{
ARTEMY VOROSHILOV, CEES OTTO,* and JAN GREVE \\ Institute for Biomedical Technology (BMTI) and Applied Optics Group, Department of Applied Physics, University of Twente, \\ P.O. Box 217, 7500 AE Enschede, The Netherlands
}

\begin{abstract}
The first application of polarization-sensitive multiplex coherent antiStokes Raman spectroscopy (MCARS) in the absence of resonance enhancement to the resolution of the secondary structure of a protein in solution is reported. Polarization MCARS spectra of bovine albumin in $D_{2} O$ were obtained in the range 1370 to $1730 \mathrm{~cm}^{-1}$ with the aid of the background suppression technique. The spectra were fitted simultaneously with a single set of parameters (band positions, bandwidths, amplitudes, and depolarization ratios). Polarized Raman spectra simulated with these parameters revealed a good correspondence with the spontaneous Raman spectra measured. The broad amide $I^{\prime}$ band was decomposed assuming the three major secondary conformations of protein, of which the contribution of $\beta$-sheet structure was found to be negligible. Relative weights of $\alpha$-helix and random coil conformations agree well with the estimates obtained with Raman and circular dichroism (CD) spectroscopies.

Index Headings: Polarization-sensitive CARS; Multichannel Raman; Conformation of proteins.
\end{abstract}

\section{INTRODUCTION}

Coherent anti-Stokes Raman spectroscopy (CARS) has proven to be a fruitful technique for studies of biophysically important molecules in condensed phase. ${ }^{1-16}$ It has become traditional to use an experimental apparatus based on narrow-band laser sources ${ }^{1,3,8,17}$ wherein the dispersion of nonlinear susceptibility is obtained by point-by-point scanning of the laser frequencies through specific Raman resonance. One of the prime concerns ${ }^{17}$ has been to obtain high-quality spectra so that the molecular information can be derived by a further CARS line-shape analysis. ${ }^{5,4,18,19}$ However, a more versatile approach involving the combination of narrow-band $\left(\omega_{\text {pump }}\right)$ and broad-band $\left(\omega_{\text {Stokes }}\right)$ semicoherent lasers, with multichannel detection has been proposed. ${ }^{20,21}$ The excitation $\omega_{\text {pump }}-\omega_{\text {Stokes }}$ then simultaneously selects all the vibrational resonances for which the frequency and phase conditions are fulfilled. Such a multiplex CARS (MCARS) technique enables rapid data acquisition and features an improved signal-tonoise ratio ${ }^{22}$ with respect to scanning methods. A decrease in signal intensity occurs though, owing to a lower spectral density of the light delivered by the broad-band source.

MCARS has commonly been used in combustion diagnostics, plasma studies, and rotational spectroscopy of gases. ${ }^{22-24}$ It has also been very successful in a number of applications in the condensed phase that included pseudoisocyanine chloride, ${ }^{2}$ rhodamine $6 \mathrm{G},{ }^{7}$ and bacteriorhodopsin. ${ }^{14,15,21}$ Picosecond MCARS provided a large amount of information on the dynamics of excited states of transstilbene ${ }^{10}$ and the isomerization of all-trans- and 9-cisretinal. ${ }^{11,12}$ In all these studies the laser excitation was chosen in the vicinity of an allowed electronic transition, and hence, the vibrationally resonant susceptibility was electronically resonant enhanced. However, to the authors' knowledge, MCARS has never been applied to nonabsorbing samples of biological macromolecules such as proteins. Applications to relatively simple compounds such as pyridine and benzene, ${ }^{2}$ cyclohexane, ${ }^{12}$ and neat $(-)-\alpha$-pinene ${ }^{25}$ have been reported.

Interference of the vibrationally resonant and the nonresonant nonlinear polarizations is a major setback to CARS in the condensed phase. The interference distorts line shapes, thus complicating the resolution of weak and/ or overlapping Raman transitions. Discrimination against the nonresonant background can be reached with the use of polarization-sensitive ${ }^{1,2,6,18}$ (PS/CARS) or phasemismatching ${ }^{4,26}$ techniques. A common feature to the above methods is the ability to control experimentally the portion of nonresonant polarization admixed into the signal. Such a heterodyning method brings an additional "dimension" into the set of spectroscopic observables. The use of heterodyning has been shown ${ }^{2,3,8}$ to heighten the sensitivity to variation of depolarization ratios. It appears to be a feasible method for resolution of broad and composite Raman bands whose substructure is insufficiently resolved with conventional spontaneous Raman (SR) spectroscopy.

Vibrational spectroscopy of proteins, both in solutions and in solid state, has received much attention in recent years, ${ }^{3,8,9,27-36}$ as it complements the data on the structure of proteins obtained with X-ray diffraction, circular dichroism (CD), and infrared spectroscopy. Major protein conformations distinguished in solution ${ }^{29,33}(\alpha$-helix, $\beta$-sheet, and random coil) exhibit Raman bands of similar origin at different frequencies. Raman estimates of the secondary structure distribution are retrieved by resolution of the amide bands which comprise an envelope of vibrations of the polypeptide backbone. The characteristic frequencies are obtained either from model proteins or from proteins with a known structure. ${ }^{27}$ Decomposition of the prominent and broad amide I band observed between 1630 and $1700 \mathrm{~cm}^{-1}$ is generally used to estimate the relative fraction of protein substructures. ${ }^{28-30,33-36}$

Polarization-sensitive CARS measurements in the offelectronically resonant case of a protein in aqueous solution were first reported for $\alpha$-chymotrypsin. ${ }^{37}$ The following application of the PS/CARS technique to a short polypeptide, lysyl-tryptophyl-lysine, in aqueous solution ${ }^{3}$ indicated that bands have a depolarization ratio notably different from that of the nonresonant background, the latter being $1 / 3$. A subsequent PS/CARS study of proteins in solutions ${ }^{8}\left(\mathrm{D}_{2} \mathrm{O}\right)$ focused on the amide $\mathrm{I}^{\prime}$ band and 


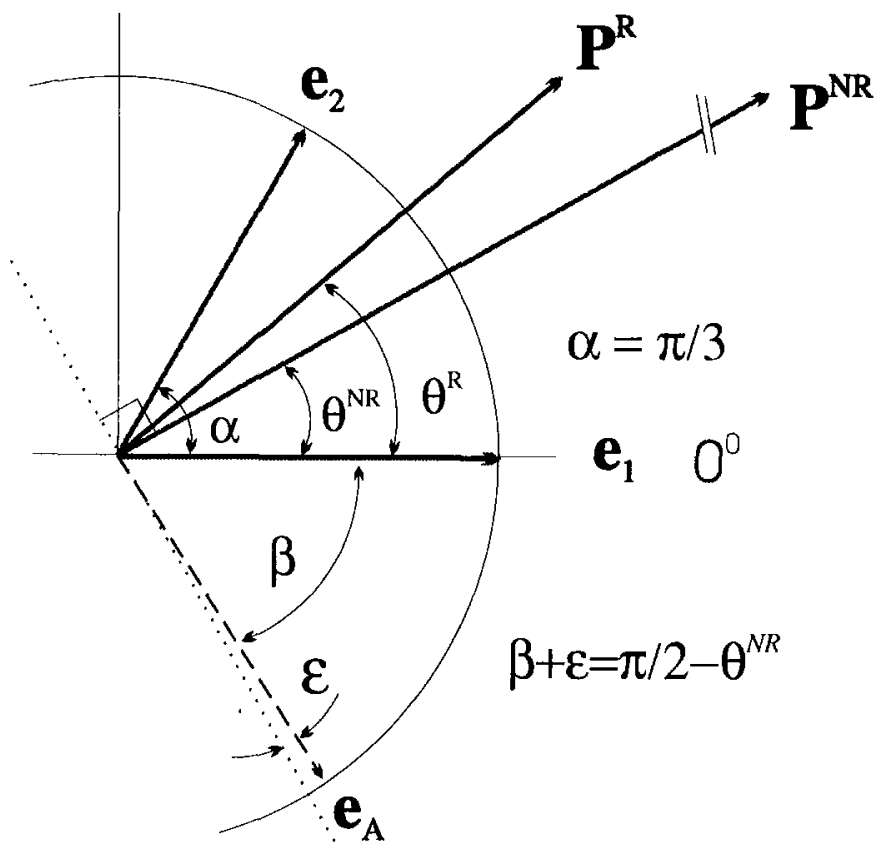

FIG. 1. Orientation of the unit polarization vectors in the frequency degenerate CARS scheme. Angles are defined relative to the direction of $\mathbf{e}_{1}$. Complete background suppression is achieved at $\epsilon=0^{\circ}$ (dotted line). Variation of $\beta$ heterodynes the resonant with the nonresonant polarization.

resolved the secondary structure components of several proteins (human albumin, $\alpha$-chymotrypsin, lysozyme, and ribonuclease A) with the aid of the heterodyning method. Estimates of the relative content of the protein conformations were based on the amplitude ratios of the amide $I^{\prime}$ constituents as obtained from line-shape fitting, and correlated well with data of X-ray, CD, and SR spectroscopies. The alteration of the protein conformation in chymotrypsin upon ligand binding (antranilic acid) was further investigated with the PS/CARS technique ${ }^{9}$ by the spectral analysis of amide I' and amide III bands and an environmentally sensitive doublet of tyrosine lines. These investigations were performed in the scanning CARS mode with the use of multimode Q-switched lasers.

In this report the design and performance are presented of a picosecond polarization-sensitive multiplex CARS spectrometer. We show that a sufficient spectral density of radiation is delivered by the picosecond dye lasers to enable PS/MCARS spectroscopy with high signal-to-noise ratio in transparent media. The methodology of MCARS combined with the heterodyning technique and their application to protein bovine albumin in solution are presented. It will be shown that the fraction of various secondary structures can be reliably determined on the basis of the polarization-sensitive resolution of the amide $\mathbf{I}^{\prime}$ band.

\section{THEORETICAL CONSIDERATIONS}

The theoretical formulation of a frequency-degenerate CARS process in its off-electron resonant case can be found elsewhere ${ }^{1,2,18,19}$ However, a concise description is provided here to outline the notations and draw the frameworks of the model used for data analysis. The intensity $I_{\text {CARS }}$ of the CARS signal field generated in a me- dium at the frequency $\omega_{\text {CARS }}=2 \omega_{1}-\omega_{2}$, and inspected through an analyzer having the transmission plane $\vec{e}_{\mathrm{A}}$, is expressed as

$$
I_{\mathrm{CARS}}\left(\omega_{\mathrm{CARS}}\right) \propto \mid\left(\left.\vec{e}_{\mathrm{A}}^{*} \cdot \vec{P}_{\mu}^{(3)}\left(\omega_{\mathrm{CARS}}\right)\right|^{2}\right.
$$

where the nonlinear polarization denoted by $\vec{P}_{\mu}^{(3)}\left(\omega_{\text {CARs }}\right)$ is induced via the third-order susceptibility $\chi_{\mu \alpha \alpha \delta}^{(3)}$ by the two incident electric fields $\vec{E}_{1}\left(\omega_{1}\right)$ and $\vec{E}_{2}\left(\omega_{2}\right)$. The following relation holds:

$$
\begin{aligned}
\vec{P}_{\mu}^{(3)}\left(\omega_{\mathrm{CARS}}\right) \propto & \chi_{\mu \alpha \alpha \delta}^{(3)}\left(\omega_{\mathrm{CARS}} ; \omega_{1}, \omega_{1},-\omega_{2}\right): \\
& E_{1}\left(\omega_{1}\right) \vec{e}_{\alpha} \cdot E_{1}\left(\omega_{1}\right) \vec{e}_{\alpha} \cdot E_{2}^{*}\left(\omega_{2}\right) \vec{e}_{\delta}^{*}
\end{aligned}
$$

where the indices $\mu, \alpha, \delta=\mathrm{x}, \mathrm{y}, \mathrm{z}$ denote the polarization of the electric fields at the frequencies $\omega_{\text {CARs }}, \omega_{1}$, and $\omega_{2}$, respectively, in Cartesian coordinates, and the asterisk ${ }^{*}$ ) marks a complex conjugate that corresponds to the emission of a photon. The total polarization $\vec{P}^{(3)}$ is generally comprised of the nonvibrational resonant polarization $\vec{P}^{(3) \mathrm{NR}}$ and the purely vibrational resonant polarization $\vec{P}^{(3) \mathrm{R}}$. In applications to solutions $\vec{P}^{(3) \mathrm{NR}}$ arises predominantly from the solvent molecules. The entire thirdorder susceptibility of such a system can be separated into different contributions, and the following representation for the observed CARS intensity is applicable:

$$
\begin{aligned}
& I_{\mathrm{CARS}}\left(\omega_{\text {CARS }}\right) \\
& \quad \propto\left|p^{\mathrm{NR}} \chi^{(3) \mathrm{NR}}+\sum_{t} p_{t}^{\mathrm{R}} \frac{\mathrm{A}_{t}^{\mathrm{R}} \exp \left(i \Theta_{t}^{\mathrm{R}}\right)}{\left(\Omega_{t}-\left(\omega_{1}-\omega_{2}\right)+i \Gamma_{t} / 2\right)}\right|^{2}
\end{aligned}
$$

where $\Omega_{t}, \Gamma_{t}, A_{t}^{\mathrm{R}}$, and $\Theta_{t}^{\mathrm{R}}$ are, respectively, the position, width, amplitude, and phase of the $t$ th Raman transition. The nonresonant background $\chi^{(3) N R}$ is here assumed to be a real value originating from the optically transparent and isotropic solvent. The surnmation in Eq. 3 runs over all $t$ vibrations with polarization factors:

$$
p^{\mathrm{R}, \mathrm{NR}}=\left[\left(1-\rho^{\mathrm{R}, \mathrm{NR}}\right)\left(\vec{e}_{\mathrm{A}}^{*} \vec{e}_{1}\right)\left(\vec{e}_{1} \vec{e}_{2}^{*}\right)+\rho^{\mathrm{R}, \mathrm{NR}}\left(\vec{e}_{\mathrm{A}}^{*} \vec{e}_{2}^{*}\right)\right],
$$

which are determined by the polarization vectors $\vec{e}_{1}$ and $\vec{e}_{2}$ of the two incident electric fields at frequencies $\omega_{1}$ and $\omega_{2}$, respectively (see Fig. 1), the transmission of the analyzer $\vec{e}_{\mathrm{A}}$, and the CARS depolarization ratios $\rho^{\mathrm{R}}$ and $\rho^{\mathrm{NR}}$.

The components of the susceptibility tensor are given by an orientational average of the contributions from individual molecules and can be expressed in terms of isotropic $\bar{\alpha}^{2}$, anisotropic $\bar{\gamma}^{2}$, and antisymmetric $\bar{\gamma}_{\mathrm{A}}^{2}$ invariants of the Raman polarizability tensor. ${ }^{5}$ In isotropic media only two of the four distinguishable components of the susceptibility tensor $\chi_{\mu \alpha \beta \delta}^{(3)}$ can be resolved by a frequency-degenerate CARS experiment, namely $\chi_{1111}^{3}$ and $\chi_{1221}^{(3)}$. The depolarization ratios are defined as $\rho^{\mathrm{R}, \mathrm{NR}}=$ $\chi_{1221}^{(3)}, \mathrm{NR} / \chi_{1111}^{(3 \mathrm{R}, \mathrm{NR}}$. The resonant CARS ratio $\rho^{\mathrm{R}}$ is related to the above invariants as follows:

$$
\rho^{\mathrm{R}}=\frac{\chi_{1221}^{(3) \mathrm{R}}}{\chi_{1111}^{(3) \mathrm{R}}}=\frac{-5 \bar{\gamma}_{A}^{2}+3 \bar{\gamma}^{2}}{45 \bar{\alpha}^{2}+4 \bar{\gamma}^{2}} .
$$

Far away from electronic transitions the Raman polarizability tensor is symmetric $\left(\bar{\gamma}_{\AA}^{2}=0\right)$. The ratio $\rho^{\mathrm{R}}$ becomes then the CARS analogue of the depolarization ratio $\rho_{\mathrm{SR}}=I_{\perp} / I_{\|}$observable with spontaneous Raman spectroscopy and $0<\rho^{\mathrm{R}}<3 / 4$. The nonresonant term in Eq. 3 originating from an optically transparent and isotropic medium is characterized by $\rho^{\mathrm{NR}}=1 / 3 .{ }^{38}$ 
In the particular polarization scheme with the angles $\alpha$ and $\beta$ defined as in Fig. 1, the polarization factors in Eq. 4 transform as follows:

$$
p^{\mathrm{R}, \mathrm{NR}}=\left[\left(1-\rho^{\mathrm{R}, \mathrm{NR}}\right) \cos \alpha \cos \beta+\rho^{\mathrm{R}, \mathrm{NR}} \cos (\alpha+\beta)\right] .
$$

Equations 3, 5, and 6 form the model for the analytical treatment of susceptibility dispersion profiles obtained experimentally.

The spatial orientation of the nonlinear polarization $\vec{P}_{t}^{(3) \mathrm{R}}$ for a particular $t$ resonance differs from the one of $\vec{P}^{(3) \mathrm{NR}}$ as determined by the magnitudes of the respective depolarization ratios:

$$
\begin{gathered}
\Theta_{t}^{\mathrm{R}}=\arctan \left(\rho_{t}^{\mathrm{R}} \cdot \tan \alpha\right) \\
\text { and } \\
\Theta^{\mathrm{NR}}=\arctan \left(\rho^{\mathrm{NR}} \cdot \tan \alpha\right) .
\end{gathered}
$$

Under nonelectron resonant conditions, the polarizations are both linearly polarized, and the nonresonant polarization is much larger than the vibrationally resonant one. The nonresonant background contribution may be suppressed by crossing the analyzer $\vec{e}_{\mathrm{A}}$ (dotted line in Fig. 1) with the direction of $\vec{P}^{(3) N R}$. The contribution of the resonant susceptibility can then be maximized by selecting the $\alpha$-angle at $\pi / 3 .{ }^{2}$ In such a case the $\vec{P}^{(3) \mathrm{NR}}$ vector is expected at $\Theta^{\mathrm{NR}}=\pi / 6$, while $\vec{P}^{(3) \mathrm{R}}$ lies at the angle $\Theta^{R}=0$ and $\Theta^{R} \approx 52.4^{\circ}$ for respectively polarized $\left(\rho^{\mathrm{R}}=0\right)$ and depolarized $\left(\rho^{\mathrm{R}}=3 / 4\right)$ vibrations. Variation in the analyzer position $\beta$ with respect to direction $\pi / 2$ - $\Theta^{\mathrm{NR}}$ admixes a portion of the background into the detected signal. The degree of heterodyning is introduced by the experimentally controlled variation of the $\epsilon$-angle.

Because of the dispersive properties of the sample, the optimal phase-match $\Delta \vec{k}=\left[2 \vec{k}_{1}\left(\omega_{1}\right)-\vec{k}_{2}\left(\omega_{2}\right)\right]-\vec{k}_{\mathrm{CARS}}\left(2 \omega_{1}\right.$ $\left.-\omega_{2}\right)=0$ cannot be achieved simultaneously for all the frequencies within the broad Stokes spectrum $\Delta \omega_{2}$, though it may be minimized within a chosen spectral range.? The frequency-dependent phase-mismatch narrows the width of an anti-Stokes spectrum in accordance with the expression

$$
I_{\mathrm{CARS}}\left(\omega_{2}\right) \propto I_{1}^{2} I_{2}\left(\omega_{2}\right) \operatorname{sinc}^{2}\left(\left|\Delta \vec{k}\left(\omega_{2}\right)\right| L / 2\right) L^{2}
$$

and limits the principally obtainable spectral range. In the narrow-band CARS experiments, the problem above is usually resolved by scanning the angle of incidence in a mechanical way to compensate for the dispersion of $\Delta \vec{k}\left(\omega_{2}\right)$. In the MCARS applications, however, the spectral distortions due to the phase-mismatch factor can be significantly reduced by shortening the sample length ${ }^{15} L$ and choosing it so that $L \ll L_{\text {Coh }}$, where $L_{\text {Coh }}=\pi /|\Delta \vec{k}|$ is the coherence length. For instance, deviations of less than $4 \%$ at the edges of a $300-\mathrm{cm}^{-1}$ broad CARS intensity profile centered at $1550 \mathrm{~cm}^{-1}$ can be expected for a $1-\mathrm{mm}$ long cuvette with water. Further, the use of tighter focusing into the sample allows one to increase the intensities $I_{1}$ and $I_{2}$ of incident beams and, therefore, to compensate for the decrease in $I_{\mathrm{CARS}}$ caused by the reduction of sample length $L$. The tighter focusing leads to an even further relaxation of phase-matching requirements ${ }^{12}$ as it expands both angular ranges with which $\vec{k}_{1}$ and $\vec{k}_{2}$ intersect.

\section{MATERIALS AND METHODS}

Bovine albumin (No A-7906) was purchased from Sig$\mathrm{ma}$ and used without further purification. The protein was dissolved in $\mathrm{D}_{2} \mathrm{O}$ to a concentration of $180 \mathrm{mg} / \mathrm{mL}$ and stored for a few hours at $+4^{\circ} \mathrm{C}$ to ensure the completion of the exchange of deuterium with amide protons. ${ }^{29}$ Heavy water instead of $\mathrm{H}_{2} \mathrm{O}$ was chosen as the solvent to avoid the interference of the protein vibrations in the 1400-1700 $\mathrm{cm}^{-1}$ Raman range with the pronounced and broad water resonance at $\sim 1640 \mathrm{~cm}^{-1}$, which shifts to $\sim 1200 \mathrm{~cm}^{-1}$ in the $\mathrm{D}_{2} \mathrm{O}$ spectrum. The stock solution was used in CARS experiments. For spontaneous Raman experiments, the solution was diluted to a concentration of $40 \mathrm{mg} / \mathrm{mL}$.

The layout of our picosecond multiplex polarization CARS spectrometer ${ }^{13}$ is based on two cavity-dumped dye lasers (3.8 MHz, Coherent 700) synchronously pumped by a mode-locked Nd:YLF laser (Antares 76-YLF, Coherent). Broad-band emission was obtained from the DCM-filled dye laser by removing the standard birefringent plate from the cavity. This semi-coherent output $(627.5-645 \mathrm{~nm})$ with 8-ps auto-correlation function (obtained with Femtochrome, FR $103 \mathrm{XL}$ ) was used as the Stokes beam. The narrow-band dye laser (Rh6G filled, 5 ps), delivering the pump beam, was tuned to $578.3 \mathrm{~nm}$ so that the Raman range 1370 to $1730 \mathrm{~cm}^{-1}$ could be covered with the excitation $\bar{\nu}_{\text {Pump }}-\Delta \bar{\nu}_{\text {stokes }}$ (see Fig. 2). Separate optical channels allowed for the adjustment of temporal overlap between pump and Stokes pulses (crosscorrelation $9.6 \mathrm{ps}$ ) and their polarization (Glan-Taylor polarizers, $\epsilon<10^{-6}$, Melles Griot). A focusing lens $(L=$ $7 \mathrm{~cm}$ ) combined the parallel beams (pulse energy $\sim 9 \mathrm{~nJ}$ each) in the sample, which was kept in a 1-mm-long cuvette. Thin $(150-\mu \mathrm{m})$ anti-reflection coated glass windows were applied to minimize stress-induced birefringence and to avoid multiple interference at the air boundaries.

The CARS continuum was collimated by a lens $(L=$ $10 \mathrm{~cm}$ ), spatially separated at the appropriate phase-match angle, analyzed with a Glan-Taylor polarizer, further attenuated, and led into a single $f / 12$ home-made polychromator. Residues of the pump and Stokes beams present in the signal beam were suppressed with holographic notch (Kaiser Optics, HNF 2090) and interference filters, respectively. To maximize dispersion on the detector, we magnified the spectral images created by the polychromator by a factor of 3 with a lens $(L=5 \mathrm{~cm})$ attached to its exit port. The spectra were detected by an imageintensified photodiode array (EG\&G 1421) and acquired by a multichannel analyzer (EG\&G 1460). The MCARS spectra were accumulated at room temperature with an exposure time of $300 \mathrm{~s}$ and read out every $0.5 \mathrm{~s}$. All spectra measured were corrected for the residual contribution of the pump beam and detector dark current. These were acquired while the Stokes beam was being blocked. Wavenumber calibration was carried out with $\mathrm{Ne}$ and $\mathrm{Ar}$ emission lamps.

The MCARS dispersion profiles are affected by (1) the spectral distribution $I_{2}\left(\omega_{2}\right)$ of the Stokes laser; (2) the extent of the phase-mismatch; (3) the wavelength-dependent response of the detection system; and (4) the individual pixel sensitivity of the multichannel detector. The 


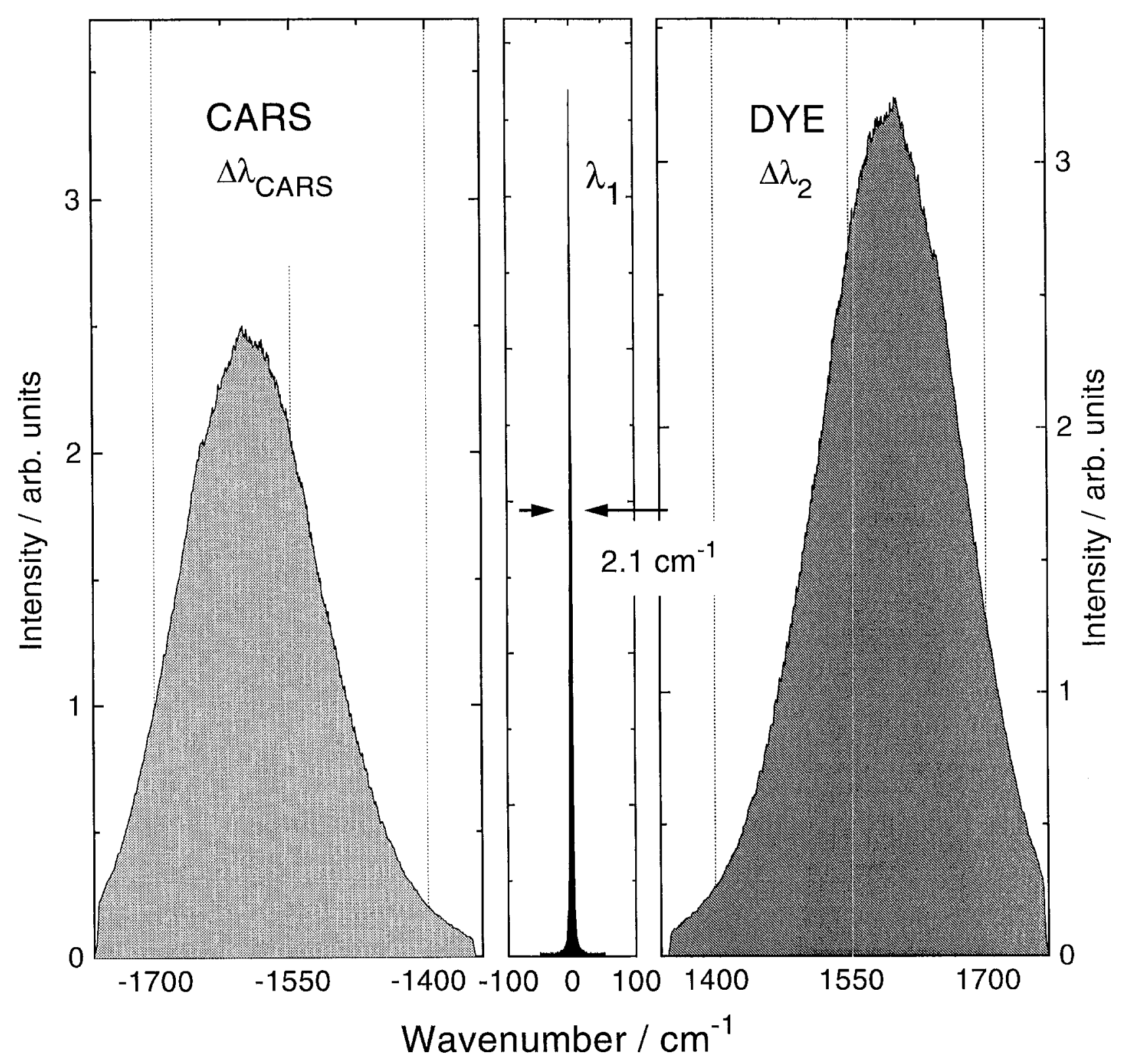

FIG. 2. Excitation scheme in the multiplex CARS experiments. Emission profiles of the broad-band Stokes laser (right) and narrow-band pump laser (center) are plotted along with the dispersion profile of the nonresonant background of $\mathrm{D}_{2} \mathrm{O}$ (left). The intensity scales differ.

MCARS spectrum of the sample and the dispersion of the nonresonant background of the solvent are obtained under the same experimental conditions. These spectra are equally influenced by the factors above. The nonresonant susceptibility of $\mathrm{D}_{2} \mathrm{O}$ shows no vibrational features within the Raman range used and is independent of the wavelength. The spectrum of the nonresonant background thus provides a convenient reference for the intensity calibration.

The MCARS spectrum shown in Fig. 2 (left) was obtained from the protein solution in $\mathrm{D}_{2} \mathrm{O}$ by setting the transmission plane of the analyzer $\beta=-\Theta^{\mathrm{NR}}=-30^{\circ}$, that is, along the theoretically predicted direction of the polarization $\vec{P}^{(3) \mathrm{NR}}$. The spectrum exhibits a width of $\sim 190 \mathrm{~cm}^{-1}$ full weight at half-maximum (FWHM), which enables MCARS coverage of an approximately $400-\mathrm{cm}^{-1}$ broad Raman range. Because of large differences in the relative magnitudes of nonresonant and resonant polarizations $\left(\chi^{(3) \mathrm{R}} / \chi^{(3) \mathrm{NR}}<10^{-2}\right)$, the spectrum in Fig. 2 shows solely the contribution of the nonresonant background. This profile was used for intensity calibration. Regular modulations in the resultant spectra, caused by unequal offset of even and odd pixels over the dynamic range of the detector, were eliminated with the use of the routine of fast Fourier transform filtering.

Spontaneous Raman spectra were obtained with a Jobin-Yvon Ramanor HG 2S spectrometer (excitation at $514.5 \mathrm{~nm}$, Spectra-Physics 2025-03) in a $90^{\circ}$ geometry. Polarized measurements were performed with the use of a sheet analyzer (Spindler \& Hoyer, $\epsilon<10^{-4}$ ) in the detection path and a $\lambda / 2$ plate in the excitation path. The VV and VH spectra of albumin solution were obtained after subtraction of the respective spectra of neat $\mathrm{D}_{2} \mathrm{O}$. Spectra were accumulated for $10 \mathrm{~s} / \mathrm{cm}^{-1}$ at $+5^{\circ} \mathrm{C}$ with 
slitwidths of $300 \mu \mathrm{m}$, corresponding to a spectral resolution of $2.5 \mathrm{~cm}^{-1}$.

\section{RESULTS AND DISCUSSION}

The resolution in the MCARS experiments is dependent on both the spectral width of the pump laser $\lambda_{1}$ and the instrumental response of spectrometer. The width of $2.1 \mathrm{~cm}^{-1}$ (FWHM) of the laser line $\lambda_{1}$ was measured independently with the Ramanor spectrometer (slits $50 \mu \mathrm{m}$, resolution $\sim 0.3 \mathrm{~cm}^{-1}$ ). The instrumental function is mainly determined by the spectral slitwidth of the polychromator $\left(1.35 \mathrm{~cm}^{-1}\right.$, as obtained from deconvolution) and the image distortions ${ }^{24}$ in the image intensifier of the detector. Since the relay lens at the exit port of the polychromator magnified the linear dispersion, the decrease in spectral resolution due to distortions in the image intensifier was minimized. The threefold expansion of the spectrum enabled us to improve the response of the image-intensified photodiode array (IPDA) from 2.2 to 0.7 $\mathrm{cm}^{-1}$. Additionally, the use of the relay lens reduced the risk that the IPDA would be saturated by the incoming photons. The laser line $\lambda_{1}$ detected in this configuration displayed an FWHM of $3.4 \mathrm{~cm}^{-1}$, which should be recognized as the actual resolution in MCARS experiments. Since the expected Raman bandwidths for the protein are typically in a range of 12 to $20 \mathrm{~cm}^{-1}$ (FWHM), ${ }^{3,32}$ the line broadening due to the finite system resolution was not further considered.

MCARS spectra of bovine albumin in $\mathrm{D}_{2} \mathrm{O}$ were resolved in the $1370-1730 \mathrm{~cm}^{-1}$ range by varying the $\epsilon$-angle over $\pm 1.5^{\circ}$ with a step of $0.5^{\circ}$ around the position of maximal background suppression. Four of the six MCARS profiles are represented in Fig. 3a-3d. Two broad bands found at 1450 and $1650 \mathrm{~cm}^{-1}$ dominate the spectra. They can, respectively, be assigned to the envelopes of $\mathrm{CH}_{2}$ and $\mathrm{CH}_{3}$ deformations and the vibrations of the deuterated polypeptide backbone (amide $I^{\prime}$ ). The substructure of the amide $I^{\prime}$ band is best seen in Fig. $3 \mathrm{~b}$ and $3 \mathrm{c}$ obtained with the least fraction of the nonresonant background. Resolution appeared possible because of a small variation in the vibrational depolarization ratio within the broad bands.

In a particular polarization scheme (Fig. 1) the polarization vectors $\vec{P}^{(3) R}$ appear between $\vec{e}_{1}$ and $\vec{P}^{(3) N R}$ for polarized bands $\left(\rho^{\mathrm{R}}<\rho^{\mathrm{NR}}\right)$ and between $\vec{P}^{(3) \mathrm{NR}}$ and $\Theta^{\mathrm{R}} \approx$ $52.4^{\circ}$ for depolarized $\left(\rho^{\mathrm{R}}>\rho^{\mathrm{NR}}\right)$ bands. An analyzer used for suppression of $\vec{P}(3)$ NR introduces a sign with which the resonant polarization $\vec{P}^{(3) R}$ contributes to the overall polarization. Since the heterodyned spectra represent the dispersion of $\chi^{\mathrm{R}}$ in the presence of a controlled amount of the nonresonant background, the sign of the dispersive contribution at the particular $\epsilon$-angle indicates the depolarization ratio of a band as compared to $\rho^{\mathrm{NR}}$. One can see that the constituents of the band at around $1450 \mathrm{~cm}^{-1}$ all have $\rho^{\mathrm{R}}>\rho^{\mathrm{NR}}$, while those of the amide $\mathrm{I}^{\prime}$ band show $\rho^{\mathrm{R}}<\rho^{\mathrm{NR}}$.

As can be seen from Fig. 3a-3d, a slight $\left(0.5^{\circ}\right)$ variation in the analyzer setting results in a drastic change of the band shapes. The heterodyning effect allows one to resolve weak vibrations at 1580 and $1603 \mathrm{~cm}^{-1}$ as well. These bands, assignable, respectively, to tryptophane and/ or phenylalanine and to tyrosine modes, ${ }^{28,31}$ both display
TABLE I. The best parameter sets as obtained from a simultaneous fit on the six polarization multiplex CARS spectra of bovine albumin in $\mathrm{D}_{2} \mathrm{O}$. The parameters are the Raman band positions, bandwidths, relative amplitudes, and depolarization ratios. Fitting parameters for the nonresonant background are $\chi^{(3) \mathrm{NR}}=1320$ a.u. and $\rho^{\mathrm{NR}}=0.304$. Heavy water bands are incorporated into the fit at 1202, 1558, 2385, and 2490 $\mathrm{cm}^{-1}$, with relative amplitudes and depolarization ratios taken from polarized spontaneous Raman measurements. A number of protein modes, such as $\mathrm{NO}_{2}$ stretching and contributions from $\mathrm{Trp}$ and $\mathrm{N}$-deuterated Trp, are introduced into the fit as a broad $\left(40-\mathrm{cm}^{-1}\right)$ band at $1329 \mathrm{~cm}^{-1}$, $\rho^{\mathrm{R}}=0.3$. Abbreviations used are His (Histidine), Phe (Phenylalanine), Trp (Tryptophan), and Tyr (Tyrosine).

\begin{tabular}{|c|c|c|c|c|}
\hline $\begin{array}{c}\frac{\Omega_{t}}{2 \pi c} \\
\left(\mathrm{~cm}^{-1}\right)\end{array}$ & $\frac{\Gamma_{t}}{2 \pi c}$ & $\begin{array}{c}A_{i}^{\mathrm{R}} \\
\text { (a.u.) }\end{array}$ & $\rho_{t}^{\mathbf{R}}$ & Assignment \\
\hline 1405 & 13 & 5.1 & 0.1 & $\begin{array}{l}\text { His: } N_{1}-C_{2}-N_{3} \text { sym. stretching } \\
N-D \text { bending }\end{array}$ \\
\hline 1421 & 16 & 10.2 & 0.08 & $\mathrm{COO}^{-}$sym. stretching. \\
\hline 1446 & 16 & 38.7 & 0.63 & $\begin{array}{l}\mathrm{C}-\mathrm{H} \text { def. overtone } \\
\mathrm{CH}_{2} \text { bending } \\
\text { Phe (?) }\end{array}$ \\
\hline 1457 & 16 & 32.0 & 0.64 & $\mathrm{CH}_{2}$ sym. def. \\
\hline 1469 & 14 & 15.3 & 0.67 & $\begin{array}{l}\mathrm{CH}_{3} \text { asym def. } \\
1477 \text { amide } \mathrm{II}^{\prime}(?)\end{array}$ \\
\hline 1584 & 11 & 9.4 & 0.73 & Phe, Trp, $N$-deuterated Trp \\
\hline 1605 & 12 & 14.3 & 0.74 & $\begin{array}{l}\text { Tyr, ring stretching } \\
\text { Phe, ring }\end{array}$ \\
\hline 1615 & 13 & 7.4 & 0.7 & $\begin{array}{l}\text { Phe, ring stretching } \\
\text { Tyr ring stretching } \\
\text { Trp (indole ring) }\end{array}$ \\
\hline 1634 & 24 & 56.5 & 0.09 & $\alpha$-Helix \\
\hline 1651 & 24 & 44.3 & 0.05 & Random coil \\
\hline 1661 & 24 & 1 & 0.0 & $\beta$-Sheet \\
\hline
\end{tabular}

$\rho^{\mathrm{R}}>\rho^{\mathrm{NR}}$. The best discrimination against the background is achieved at $\beta \approx 62.5^{\circ}$. The efficiency of the suppression was judged by observing the minimal background contribution in the range of 1500 to $1550 \mathrm{~cm}^{-1}$ and at the spectral edges where no vibrations were expected. The exact value of the angle $\Theta^{\mathrm{NR}}$ and, thus, the depolarization ratio $\rho^{\mathrm{NR}}$ could only be obtained by fitting the MCARS spectra.

In accordance with the above model, the six PS/MCARS spectra were fitted simultaneously with a single set of vibrational parameters including band positions, bandwidths, amplitudes, and depolarization ratios. In the fit, the vibrational phases were all assumed to be zero. It appeared necessary to incorporate in the fitting twelve protein modes with known frequencies and assignments. ${ }^{27,28,31}$ The fit quality was judged by the minimal achievable value of the chi-square function weighted with reciprocal experimental data. The best parameter set obtained is given in Table I along with the band assignments. The corresponding curves are plotted in Fig. 3a-3d in bold. Four solvent modes were also introduced with vibrational parameters as obtained from a fit of the polarized SR spectra of $\mathrm{D}_{2} \mathrm{O}$. In fitting the amide $\mathrm{I}^{\prime}$ region only $\alpha$-helix, $\beta$-sheet, and undefined (random coil) conformations ${ }^{29,33}$ were considered. The amount of respective fractions can be estimated from the amide spectral intensities (band areas ${ }^{33}$ ). Representing the amide bands as linear combinations is allowed under the assumption that the Raman polarizability tensor is nearly constant for different structure types. The amide I band arises primarily because of the stretching vibrations of the carbonyl group. ${ }^{28}$ Deuteration of the polypeptide 


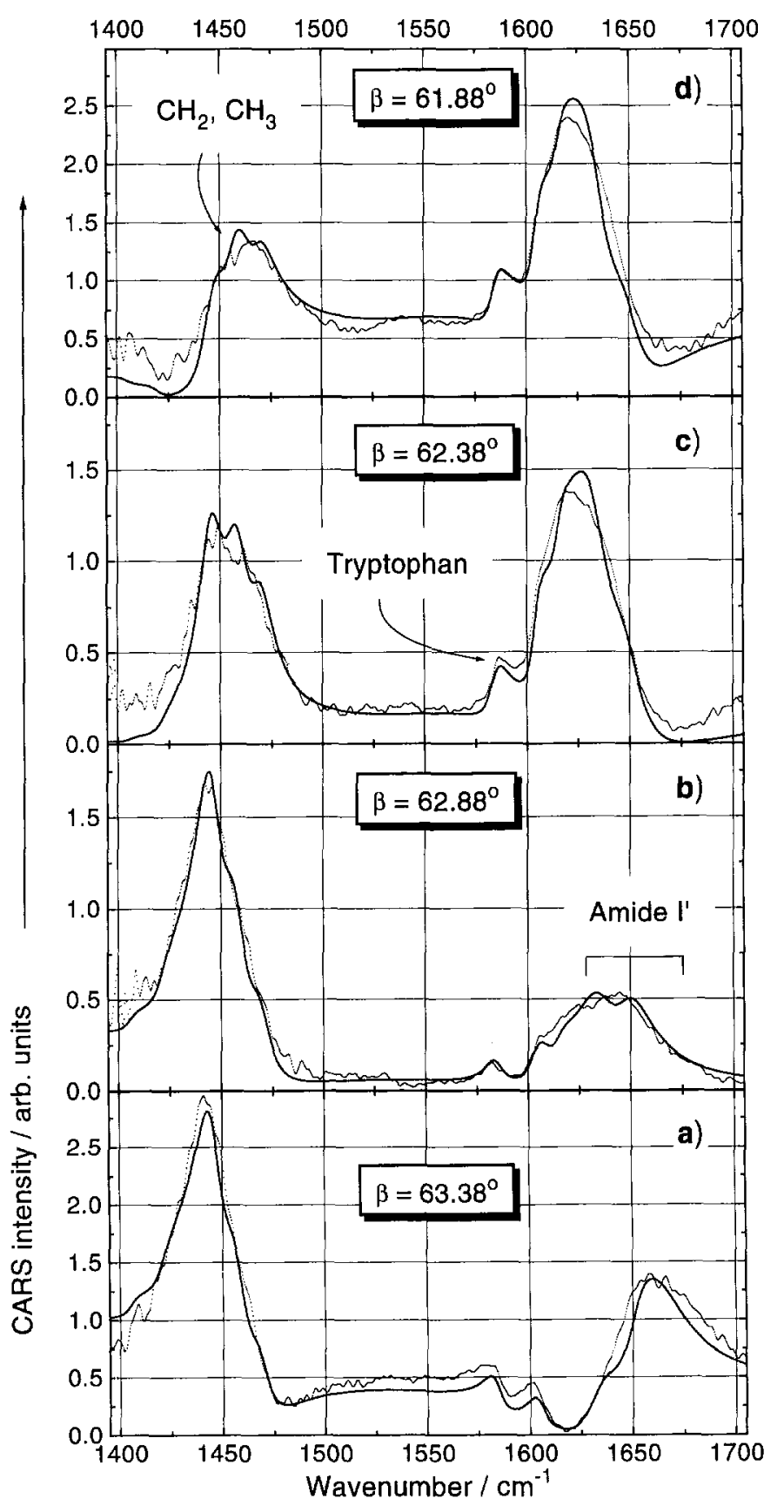

A. Voroshilov et al., "Secondary Structure of Bovine Albumin as Studied by Polarisation Sensitive Multiplex CARS Spectroscopy"

Fig. 3. Multiplex CARS spectra of bovine albumin in $\mathrm{D}_{2} \mathrm{O}$ (dotted lines), $\alpha=60^{\circ} 10^{\prime}$. From a to d, $\beta$ is varied from $63.38^{\circ}$ to $61.88^{\circ}$ with steps of $0.5^{\circ}$ as indicated by the insets. Spectra are normalized on the nonresonant background profile of the solvent and FFT filtered. Curves in bold are the best simultaneous fits with parameters listed in Table $\mathbf{I}$. Note the change of CARS intensity scale in going from a to $\mathbf{d}$.

backbone leads to a small overall downshift $\left(2-12 \mathrm{~cm}^{-1}\right.$, Ref. 31) of the amide I frequency. As shown in Ref. 27 for the model poly-L-lysine, upon deuteration, the three conformations display Raman peaks at, respectively, 1632, 1658 , and $1660 \mathrm{~cm}^{-1}$. These Raman positions were introduced into the fit as initial parameters. The Raman bandwidth of each contributor was taken to be equal.
As can be seen in Fig. 3a-3d, the spectral features are well reproduced by the curves simulated. The noise contribution in the MCARS spectra, which can be estimated to be within $6 \%$, limits the accuracy of the fits. The noise arises $^{23}$ from (1) pulse-to-pulse variations in the spectral energy of the Stokes dye laser, (2) pulse-to-pulse variations in the spatial and temporal overlap of the pump and Stokes pulses, and (3) shot noise of the detector. Since picosecond lasers with a high repetition rate $(3.8 \mathrm{MHz})$ were used, the influence of the first two factors was minimized by accumulating the signal for some $10^{9}$ laser pulses. The detector noise, though normally negligible, became dominant at very low CARS intensities, such as at the edges of spectra in Fig. 3a-3d. The fit quality is also dependent on the signal-to-nonresonant background ratio. A fraction of the background emerges even in "background-free" spectra owing to the stress-induced birefringence of cuvette windows. Such a residual background was experimentally minimized by introducing an opposed ellipticity in the polarization of the Stokes beam, the latter arising upon reflection at the matching mirror. ${ }^{3}$

The calculated nonresonant depolarization ratio $\rho^{\mathrm{NR}}=$ 0.304 differs from its expectation value of $1 / 3$, indicating a slightly elliptical polarization of the nonresonant contribution. This result can be attributed to the contribution of the electronically resonant susceptibility of protein, which arises because of the two-photon absorption. The determination of the $\rho_{t}^{\mathrm{R}}$ values (and the corresponding amplitudes $A_{t}^{\mathrm{R}}$ ) from the set of background-suppressed spectra is most accurate for those vibrations which have $\rho_{t}^{\mathrm{R}}$ close to $\rho^{\mathrm{NR}}$, as the heterodyning effect enables one to control their shape most efficiently. On the other hand, the heterodyning technique fails to resolve the modes with $\rho_{t}^{\mathrm{R}} \cong \rho^{\mathrm{NR}}$. There have been attempts ${ }^{3,8}$ to fit polarization CARS spectra of various proteins on the basis of the assumption that protein bands are either purely polarized $\rho_{t}^{\mathrm{R}}=0$ or depolarized $\rho_{t}^{\mathrm{R}}=0.75$. In Ref. 8 the authors decomposed the amide $I^{\prime}$ band with the three subelements assuming equal polarization properties (all $\rho_{t}^{\mathrm{R}}$ taken to be zero). Such an approach, though, appears to be inconsistent with the motivation of using the PS/CARS technique because of its unique sensitivity to the variations of $\rho_{t}^{\mathrm{R}}$ ratios. Accurate determination of the depolarization ratios was limited ${ }^{8}$ by significant pulse-to-pulse fluctuations $(\sim 30 \%)$ and was possible only for the $0.2-0.4$ range.

Because of the higher spectral quality in multiplex experiments as compared to scanning PS/CARS, the spectra with smaller signal-to-background ratio can still be resolved. This capability permits one to enlarge the range of $\epsilon$-angle variations (up to $\pm 2^{\circ}$ in the given case) and, therefore, to make better use of the heterodyning technique. The latter enhances the accuracy with which the parameters are obtained from the simultaneous fitting of the set of spectra. The estimated fitting accuracy is $\sim 1.5$ $\mathrm{cm}^{-1}$ for the band positions and is $\sim 8 \%$ for the bandwidths. One can see from Fig. $3 a-3 d$ and Table $I$ that, for the strongest bands, such as those at 1634 and 1651 $\mathrm{cm}^{-1}$, the magnitude of $\rho_{t}^{\mathrm{R}}$ can be established with an accuracy of up to 0.05 , thanks to the high spectral quality achieved with the multiplex PS/CARS. The validity of our fits was verified by simulating the polarized Raman spectra of albumin with the best set of MCARS parameters. The simulated profiles are plotted in Fig. $4 \mathrm{a}$ and 

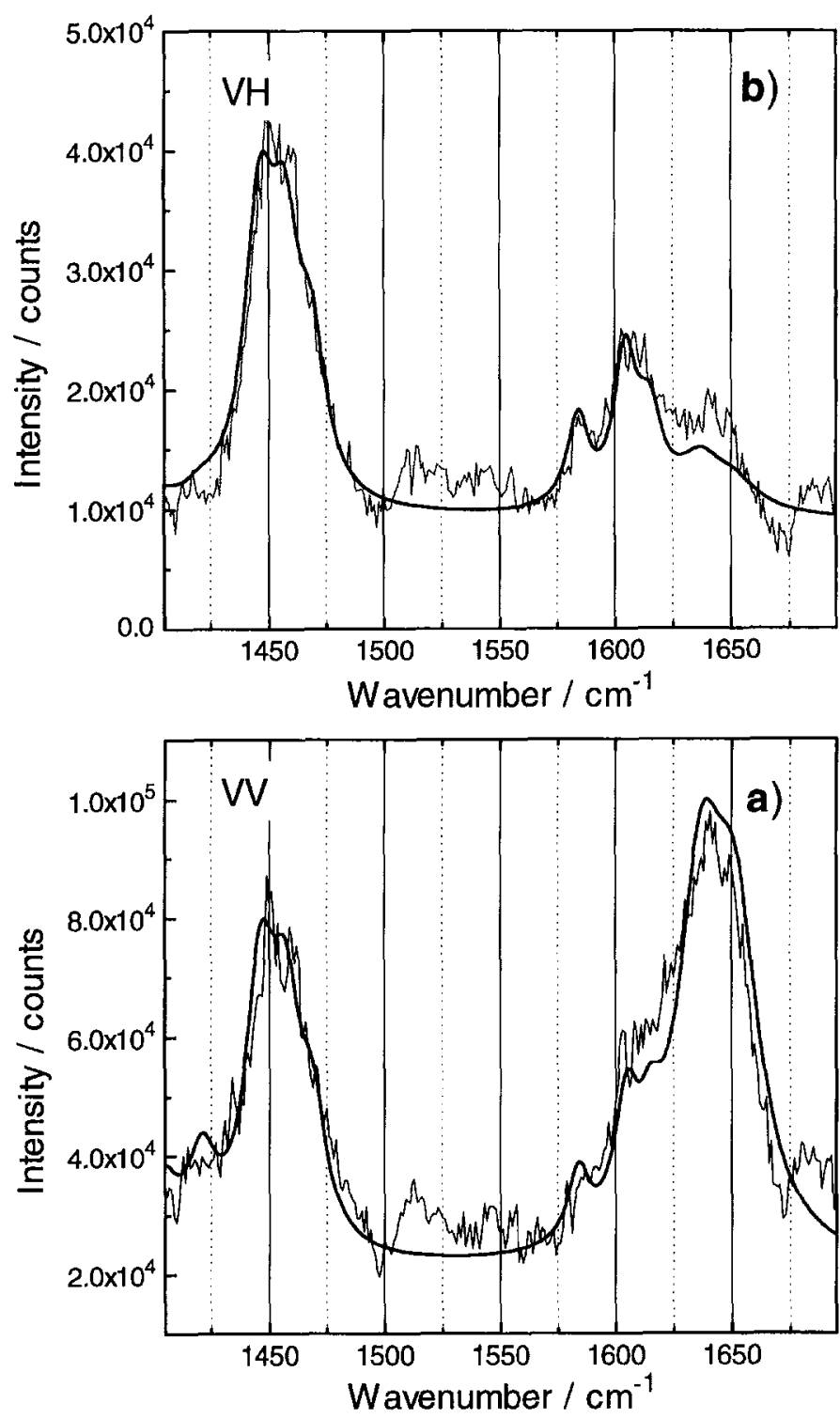

FIG. 4. Polarized spontaneous Raman spectra of bovine albumin in $\mathrm{D}_{2} \mathrm{O}(40 \mathrm{mg} / \mathrm{mL}$, background-subtracted) and corresponding spectra (in bold) reconstructed from the best set of CARS fitting parameters.

$4 \mathrm{~b}$ together with the VV and VH spontaneous Raman spectra observed experimentally. Figure 4 shows a good correspondence between the curves obtained independently. The discrepancy, however, remains in the amplitude/depolarization ratio calculated for the relatively weak vibrations at 1421 and $1615 \mathrm{~cm}^{-1}$.

As can be seen from Table I, a negligible amplitude of the mode at $1661 \mathrm{~cm}^{-1}$, corresponding to $\beta$-sheet conformation, was calculated. This result agrees well with the data reported for bovine albumin. ${ }^{8,28,29}$ Figure 5 shows the relative fractions of the albumin substructures as calculated from the CARS fit parameters. Estimates obtained with other methods are given in Fig. 5 as well. Comparing the relative content of the conformations evaluated by scanning PS/CARS for human albumin 8 (52\% helical conformation vs. $47 \%$ of random coil) and the results of our PS/MCARS on bovine albumin (respectively $56 \%$ and $44 \%$ ), it should be mentioned that, in Ref. 8, the estimates were made by comparing the

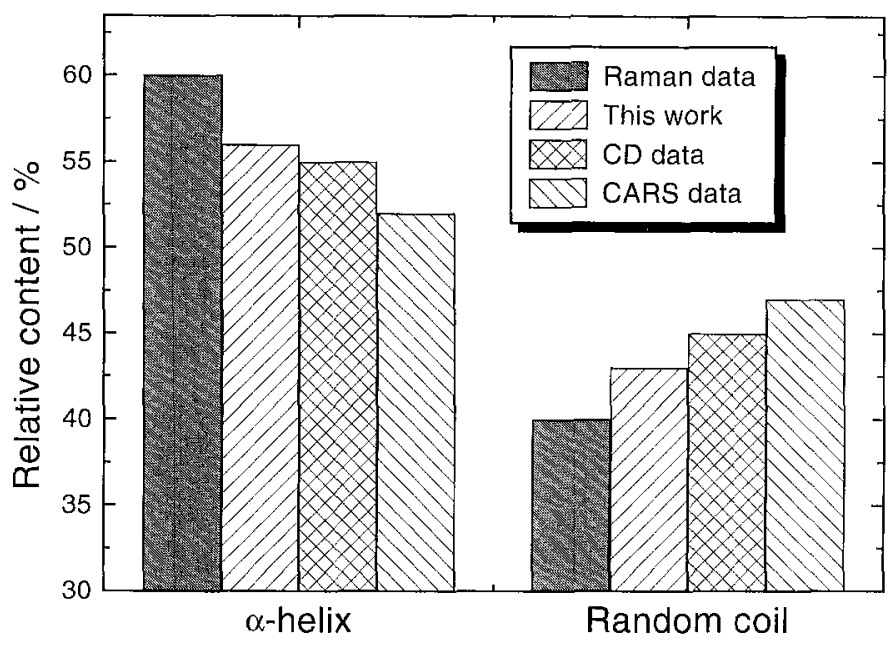

Fig. 5. Comparison between estimates of the relative content of albumin secondary structures obtained with multiplex PS/CARS (this work), spontaneous $\operatorname{Raman}^{29}$ spectroscopy, circular dichroism, ${ }^{28}$ and polarization-sensitive scanning CARS. ${ }^{8}$

amplitude ratios of amide $\mathrm{I}^{\prime}$ contributors, instead of integrated areas. Inspection of Fig. 5 reveals reasonable correspondence of PS/MCARS data to the results obtained with $C D$ and Raman spectroscopy. It shows that multiplex PS/CARS is capable of yielding sufficiently accurate estimates of the protein structural subelements.

\section{CONCLUSION}

A picosecond multiplex CARS spectrometer has been developed enabling the acquisition of $\sim 400 \mathrm{~cm}^{-1}$ broad spectra with spectral resolution of $3.4 \mathrm{~cm}^{-1}$ and high polarization accuracy. An improvement in signal-to-noise ratio, as compared to that with scanning techniques, is achieved by exploiting the statistically superior broadband CARS excitation and detection, and averaging over some $10^{9}$ excitation events.

It has been demonstrated that background-free MCARS spectra of transparent samples can be obtained. Spectra of protein bovine albumin in $\mathrm{D}_{2} \mathrm{O}$ have been resolved in the $1370-1730 \mathrm{~cm}^{-1}$ Raman range and fitted simultaneously with a single set of vibrational parameters. Major bands have been assigned to protein backbone modes (amide $\mathrm{I}^{\prime}$ ) and $\mathrm{CH}_{2}, \mathrm{CH}_{3}$ deformations. The amide $\mathrm{I}^{\prime}$ band has been decomposed into the contributions of the protein's secondary structures for which the relative content was determined. Relative weights of $\alpha$-helix and random coil conformations ( 56 to $44 \%$ ) agree reasonably well with Raman and CD data available.

\section{ACKNOWLEDGMENTS}

The authors wish to thank Dr. B. Toleutaev for his valuable contribution to the design of spectrometer and for stimulating discussions. The experimental contribution of J. Kanger is also gratefully acknowledged.

1. S. A. Akhmanov and N. I. Koroteev, Methods of Nonlinear Optics in Light Scattering Spectroscopy (Nauka, Moscow, 1981), in Russian.

2. R. Brakel, V. Mudogo, and F. W. Schneider, J. Chem. Phys. 84, 2451 (1986). 
3. T. A. H. M. Scholten, G. W. Lucassen, F. F. M. de Mul, and J. Greve, Opt. Comm. 5, 328 (1989).

4. G. W. Lucassen, W. P. de Boej, and J. Greve, J. Raman Spectrosc. 24, 383 (1993).

5. R. Igarashi, Y. Adachi, and S. Maeda, J. Chem. Phys. 72, 4308 (1980).

6. P. A. Apanasevich, V. V. Kvach, V. P. Kozich, and V. A. Orlovich, Appl. Spectrosc. 38, 699 (1983)

7. V. S. Adams, L. A. Carreira, and L. Azarraga, Appl. Spectrosc. 38, 861 (1984).

8. A. Yu. Chikishev, G. W. Lucassen, N. I. Koroteev, C. Otto, and J. Greve, Biophysical J. 63, 976 (1992).

9. A. Yu. Chikishev, N. I. Koroteev, C. Otto, and J. Greve, Proc. SPIE 2370, 140 (1994).

10. S. A. Payn and R. M. Hochstrasser, J. Phys. Chem. 90, 2068 (1986).

11. T. Tahara, B. N. Toleutaev, and H. Hamaguchi, J. Chem. Phys. 100, 786 (1994).

12. B. N. Toleutaev, T. Tahara, and H. Hamaguchi, Appl. Phys. B 59, 369 (1994).

13. B. N. Toleutaev, J. S. Kanger, C. Otto, and J. Greve, "Scanning Multichannel CARS Technique for Improved Low-Noise Measurements", in Proceedings of the XIV International Conference on Raman Spectroscopy, N.-T. Yu and X.-Y. Li, Eds. (Wiley, New York, 1994), pp. 442-443.

14. G. N. Atkinson, "Picosecond Time-Resolved Coherent Raman Scattering from Biophysical Systems", in Proceedings of the XIII International Conference on Raman Spectroscopy, W. Kiefer, Ed. (Wiley, New York, 1992), pp. 192-193.

15. L. Ujj, B. L. Volodin, A. Popp, J. K. Delaney, and G. N. Atkinson, Chem. Phys. 182, 291 (1994).

16. A. Voroshilov, G. W. Lucassen, C. Otto, and J. Greve, J. Raman Spectrosc. 26, 443 (1995).

17. T. A. H. M. Scholten, G. W. Lucassen, and F. F. M. de Mul, J. Greve, Appl. Opt. 27, 3225 (1988).

18. S. A. Akhmanov, A. F. Bunkin, S. G. Ivanov, and N. I. Koroteev, Sov. Phys. JETP 47, 667 (1978).

19. Y. R. Shen, The Principles of Nonlinear Optics (Wiley, New York, 1984).

20. W. B. Roh, P. W. Schneider, and J. P. E. Taran, Appl. Phys. Lett. 29, 174 (1976).

21. J. Tretzel and F. W. Schneider, Chem. Phys. Lett. 66, 475 (1979).

22. D. A. Greenhalgh, "Quantitative CARS Spectroscopy", in Advances in Non-Linear Spectroscopy, R. J. H. Clark and R. E. Hester, Eds. (Wiley, New York, 1988), Vol. 15, pp. 193-251.

23. D. R. Snelling, R. A. Sawchuk, and R. E. Mueller, Appl. Opt. 24, 2771 (1985)

24. D. J. Rakestraw, R. P. Lucht, and T. Dreiner, Appl. Opt. 28, 4116 (1989).

25. R. Brakel, V. Mudogo, and F. W. Schneider, "Polarization CARS Spectroscopy: General Aspects and Application to Optically Active and Absorbing Media", in Proceedings of the European Conference on Spectroscopy of Biological Molecules, A. J. P. Alix, L. Bernard, and M. Manfeit, Eds. (Wiley, New York, 1985), pp. 42-44.

26. T. A. H. M. Scholten, G. W. Lucassen, F. F. M. de Mul, and J. Greve, Appl. Opt. 28, 1387 (1989).

27. T. J. Yu, J. L. Lippert, and W. L. Peticolas, Biopolymers 12, 2161 (1973).

28. B. G. Frushour and J. L. Koenig, "Raman Spectroscopy of Proteins", in Advances in Infrared and Raman Spectroscopy, R. J. H. Clark and R. E. Hester, Eds. (Heydon, London, 1975), Vol. 1, pp. $35-97$.

29. J. L. Lippert, D. Tyminski, and P. J. Desmeules, J. Am. Chem. Soc. 98, 7075 (1976).

30. R. W. Williams and A. K. Dunker, J. Mol. Biol. 152, 783 (1981).

31. I. Harada and H. Takeuchi, "Raman and Ultraviolet Resonance Raman Spectra of Proteins and Related Compounds", in Spectroscopy of Biological Systems, R. J. H. Clark and R. E. Hester, Eds. (Wiley, New York, 1986), Vol. 13, pp. 113-175.

32. D. M. Byler and H. Susi, Biopolymers 25, 469 (1986).

33. M. Berjot, J. Marx, and A. J. P. Alix, J. Raman Spectrosc. 18, 289 (1987).

34. C. Otto, F. F. M. de Mul, and J. Greve, Biopolymers 26, 1667 (1987).

35. C. Otto, F. F. M. de Mul, B. J. M. Harmsen, and J. Greve, Nucleic Acids Research 15, 7605 (1987).

36. J. C. Sanders, P. I. Haris, D. Chapman, C. Otto, and M. A. Hemminga, Biochemistry 32, 12,446 (1993).

37. A. Yu Chikishev, "Application of Spontaneous Raman and CARS Spectroscopy to the Study of Enzyme Action", in Proceedings of the International Conference on Laser Scattering Spectroscopy of Biological Objects, J. Stepanek, P. Anzenbacher, and B. Sedlacek, Eds. (Elsevier, Amsterdam, 1987), pp. 309-316.

38. D. A. Kleinman, Phys. Rev. 126, 1977 (1962). 